\title{
Can Observational Gait Assessment Tools be used to Assess Independent Walking in Stroke Patients?
}

\author{
Sung-Kwang Ju, PT, $\mathrm{PhD}^{\dagger}$
}

Department of Physical Therapy, College of Biomedical Science, Kaya University, Gimhae, Korea

Received: November 12021 / Revised: November 32021 / Accepted: November 272021

(c) 2022 J Korean Soc Phys Med

\section{| Abstract |}

PURPOSE: A gait assessment is an important component of the rehabilitation process, and observational gait assessment (OGA) is used routinely in clinical settings. This study examined the association of OGA tools with the independent walking ability in stroke patients to determine a cutoff value of the OGA tool according to independence levels of stroke patient gait.

METHODS: Two hundred ten hemiparetic stroke patients participated in the study. The independence of gait was identified using the Functional Ambulation Category (FAC) classifications. The walking ability was assessed using OGA tools (Rivermead Visual Gait Assessment [RVGA], Wisconsin Gait Scale [WGS], Tinetti Gait Scale [TGS], and Functional Gait Analysis [FGA]).

RESULTS: Stepwise multiple regression analysis showed that among the OGA tools, the FGA correlated with the FAC. The FGA explained approximately $77 \%$ of the variance in

†Corresponding Author : Sung-Kwang Ju

jskwang84@hanmail.net, https://orcid.org/0000-0003-3125-812X This is an Open Access article distributed under the terms of the Creative Commons Attribution Non-Commercial License (http://creativecommons.org/licenses/by-nc/3.0) which permits unrestricted non-commercial use, distribution, and reproduction in any medium, provided the original work is properly cited.
FAC. In distinguishing the independence levels, the cutoff values were as follows: between FAC 1 and FAC 0 was .5 points; between FAC 2 and lower levels, 5.5; between FAC 3 and lower levels, 11.5; between FAC 4 and lower levels, 14.5; and between FAC 5 and lower levels, 18.5. Items 1, 2, 3 , and 10 were identified as explaining most of the variance in the FGA in the stepwise multiple regression.

CONCLUSION: The present study found that the FGA is an assessment tool related to the level of gait independence after stroke. Furthermore, the FGA total score can serve as an index of the increase in independence level after stroke.

Key Words: Gait, Observation, Outcome assessment, Stroke

\section{Introduction}

The impairment of walking ability is one of the most important determinants of disability in stroke patients [1]. After a stroke, the gait speed and cadence decrease, while gait cycle duration and double limb support time increase; the paretic limb has a longer swing phase and a shorter stance phase than the contralateral limb [2]. The effects of these changes on global walking performance are remarkable. A previous study found that $40 \%$ of patients require assistance with indoor ambulation at three weeks after stroke, and 15\% still require assistance at six months [3]. Another study 
reported that $82 \%$ of patients had not fully recovered community ambulation by three months or more after stroke [4]. Thus, gait recovery may improve the overall functioning and wellbeing and is a key goal in stroke rehabilitation [5,6], walking ability, with or without physical assistance or supervision for discharge planning [7]. Therefore, appropriate assessment and treatment of gait are crucial [8].

Gait assessment is an important component of the rehabilitation process [9]. For a gait assessment, 3-dimensional computerized analysis is a criterion standard because it provides objective data on electromyography and kinetic and spatiotemporal parameters [10]. Nevertheless, it requires custom instrumentation [10], takes a longer time, and it is not always available for clinical practice. Therefore, the most commonly used methods in the therapeutic environment are clinical tests and scales [11, 12]. On the other hand, observational gait analysis is inexpensive and requires little or no technology. Furthermore, observational measures of coordinated gait components are readily available [9]. Given that observational gait analysis is currently widely used, it is important to choose the optimal tool for the analysis [13]. Observational gait analysis is the most common approach for estimating the gait kinematics and deviations from normal gait patterns [14,15]. GorGarcía-Fogeda et al. [13] reported that the Wisconsin Gait Scale (WGS), Rivermead Visual Gait Analysis (RVGA), and Tinetti Gait Scale (TGS) are valid and reliable for assessing the deviations from the gait patterns in stroke patients. The Functional Gait Assessment (FGA) [16] is a modification of the Dynamic Gait Index (DGI) that was developed to improve the reliability of the DGI and reduce the ceiling effect observed with the DGI in patients [17].

The independence of gait is often measured using the Functional Ambulation Category (FAC), which was designed to provide information on the level of physical support required by patients to ambulate safely [18]. The FAC is reliable and valid for classifying a hemiplegic gait. The FAC allows a rapid visual assessment of walking; it is simple to use, easy to interpret, and cost-effective [19]. Thus, the FAC is a useful routine clinical assessment tool that can be helpful for research purposes to measure the walking outcome [20]. On the other hand, the FAC may lack precision and exhibit a ceiling effect [21, 22].

Many studies examining the interventions for gait in stroke patients have used the walking progress as an evaluation criterion [7, 8, 13]. Studies on the assessment tools for evaluating independent gait in stroke patients are lacking. Hence, this study examined whether the clinical tools currently used for assessing gait independence in stroke patients can be used effectively for assessing independent gait. Thus, the aims of this study were as follows: (1) to investigate which observational gait assessment (OGA) tool best measures the independence of gait, and (2) to suggest cutoff values for these tools according to the level of gait independence among stroke patients.

\section{Methods}

\section{Participants}

This study was conducted between November 2015 and July 2016. The data for this study were obtained from the records stored in the $\mathrm{K}$ rehabilitation hospital database. Two hundred and ten hemiparetic stroke patients participated in this study. A power analysis was performed with $\mathrm{G}^{*}$ power software ver. 3.1.9.2 (Franz Faul, University of Kiel, Kiel, Germany). The sample size was calculated with a power of .80 , alpha level of .05 , effect size of .15 , and number of predictors of 20 . Hence, a sample size of 157 participants was deemed necessary for multiple linear regression analysis. The inclusion criteria were age over 18 years, diagnosis of cerebral hemorrhage or cerebral infarction determined based on the magnetic resonance imaging findings, hemiparetic symptoms, inability to walk or impaired walking capacity, and ability to understand verbal and written information during the testing procedures. Subjects were excluded if they had any comorbid disability 
unrelated to the stroke, or any medical, musculoskeletal, cardiovascular, or other neurological disorders. Patients with mental problems, skin problems, such as pressure ulcers, and pain related to stroke, were excluded. All subjects involved rehabilitation program provided written informed consent before participation. Ethical approval for this study was obtained from the Inje University Faculty of Health Sciences Human Ethics Committee (2015296-4).

\section{Procedures}

The level of gait independence of all the participants was defined based on the FAC. The FAC is a reliable and valid assessment instrument comprised of six categories designed to provide information on the level of physical support needed by patients to ambulate. A FAC of 0,1 , $2,3,4$, and 5 indicates nonfunctional ambulator, dependent on physical assistance (level 2, continuous manual contact), dependent on physical assistance (level 1, intermittent touch), dependent on supervision, independent and level surface only, independent and everywhere, respectively [18]. The gait ability of each participant was assessed by a therapist using OGA tools, including the RVGA, WGS, TGS, and FGA. The therapist observed and evaluated the gait of each patient according to the OGA tools.

\section{Outcome assessment}

1) Rivermead Visual Gait Assessment (RVGA)

The RVGA involves two observations of the arms, covering the gait swing and stance, and 18 observations of the trunk and lower limbs (11 during the stance phase and seven during the swing phase of gait). These observations consider only one side at a time. A four-point scale was used to quantify the degree of abnormality for each of the component items: $0=$ normal, $1=$ mild, $2=$ moderate, and $3=$ severe abnormality. A global score can be calculated by summing the deviation scores, with the total ranging from 0 (normal gait) to 59 (grossly abnormal gait) [23].
2) Wisconsin Gait Scale (WGS)

The WGS consisted of 14 assessment criteria representing the clinically relevant components of gait. The criteria pertained to the pattern of body movements in each gait phase. The items were grouped into four phases: stance phase, toe-off, swing phase, and heel strike of the affected leg. Each item was scored from 1 (normal) to 3 (pathological), except for items 1 (use of a hand-held gait aid), which was scored from 1 to 5 , and 11 (knee flexion from toe-off to mid-swing), which was scored from 1 to 4. The gait parameters were scored relative to the unaffected side or the gait parameters of healthy subjects. A higher score indicates a more seriously affected gait [24].

\section{3) Tinetti Gait Scale (TGS)}

The TGS consists of eight items assessing the following: deficits in coordinated gait components (two items), compensatory strategies (five items), and temporal aspects of gait (one item). Six items were scored 0 (deviation) or 1 (normal), and two were scored as 0, 1 (deviation), or 2 (normal). The overall scores on the TGS ranged from 0 (highest deviation) to 12 points (normal). The TGS was originally developed to identify an abnormal gait and took 5 minutes to score [25].

\section{4) Functional Gait Analysis (FGA)}

The FGA consisted of 10 tasks, each scored on a four-point (0-3) ordinal scale for a maximum possible total score of 30. The FGA items included gait on a level surface, change in gait speed, gait with horizontal and vertical head turns, gait with a pivot turn, gait when stepping over an obstacle, gait with a narrow base of support, gait with the eyes closed, ambulating backward, and navigating steps. The FGA is easy to administer in most clinical settings [16].

\section{Statistical Analysis}

Statistical analysis was performed using PASW software (ver. 22.0; SPSS, Inc., Chicago, IL, USA). The descriptive 
statistics were calculated for RVGA, WGS, TGS, and FGA. The Spearman's correlation coefficients (r) were calculated to examine the potential multicollinearity among these tools. Multivariable linear regression models were applied to the results of all tools (i.e., RVGA, WGS, TGS, and FGA) used to examine the affected tools to the independence of gait of the stroke patients. Multivariable linear regression was applied to examine the sub-items of each assessment tool associated with independent walking. Moreover, the receiver operating characteristic (ROC) curves were calculated to determine the cutoff value for each tool, i.e., the FAC level at which the optimal sensitivity and specificity were achieved.

\section{Results}

\section{Participant characteristics}

This study included 210 stroke patients with a mean age of 58.3 years. The scores on the FAC were as follows: 0 points, 30 participants; 1 point, 32 participants; 2 points, 32 participants; 3 points, 32 participants; 4 points, 40 participants; and 5 points, 44 participants. Table 1 lists the characteristics of the study participants (Table 1).

\section{Spearman's correlation}

The FAC scores were positively correlated with the TGS $(r=.781)$ and FGA $(r=.884)$ scores and negatively correlated with the RVGA $(r=-.703)$ and WGS $(r=-.798)$ scores (Table 2).

\section{Stepwise multiple linear regression on the OGA}

The only observational tool included in the stepwise multiple regression was the FGA. A large amount ( $77 \%)$ of the variance in the FAC scores could be explained by the FGA scores $(\mathrm{B}=.880, \mathrm{p}=.000)$ (Table 3). The WGS $(\mathrm{p}=.684)$, TGS $(\mathrm{p}=.879)$, and RVGA $(\mathrm{p}=.773)$ were excluded by stepwise regression analysis.
Table 1. General Characteristics of the Participants

$(\mathrm{N}=210)$

\begin{tabular}{lc}
\hline \multicolumn{1}{c}{ Variables } \\
\hline Age (years) & $58.3 \pm 13.1$ \\
Height $(\mathrm{cm})$ & $165.9 \pm 7.8$ \\
Body weight $(\mathrm{kg})$ & $63.4 \pm 11.2$ \\
Hemiparetic side & \\
Left & 100 \\
Right & 110 \\
Types of damage & 115 \\
Infarction & 95 \\
Hemorrhage & $15.3 \pm 13.9$ \\
\hline
\end{tabular}

Values are mean $\pm \mathrm{SD}$ or number.

Table 2. Spearman Correlations between the FAC and OGA Tools

\begin{tabular}{cccccc}
\hline & WGS & TGS & RVGA & FGA & p-Value \\
\hline FAC & -.787 & .774 & -.690 & .880 & .000 \\
\hline
\end{tabular}

FAC: Functional Ambulation Category; OGA: Observational Gait Assessment; WGS: Wisconsin Gait Scale; TGS: Tinetti Gait Scale; RVGA: Rivermead Visual Asessment; FGA: Functional Gait Analysis

Table 3. Stepwise Linear Regression Analysis of the OGA Tools

\begin{tabular}{cccccc}
\hline Source & $\mathrm{R} 2$ & Beta & $\mathrm{t}$ & $\mathrm{p}$-Value & $\mathrm{VIF}$ \\
\hline Overall model & .77 & & & & \\
FGA & & .880 & 24.695 & .000 & 1.000 \\
\hline
\end{tabular}

OGA: Observational Gait Assessment; FGA: Functional Gait Analysis; VIF: Variance Inflation Factor.

\section{Cutoff values}

Table 4 lists the cutoff values for FGA scores by FAC category. In distinguishing among independence levels, the cutoff values were as follows: between FAC 1 and FAC 0 was .5 points ( $\mathrm{AUC}=.953 ; 95 \% \mathrm{CI}, .926-.980)$; between FAC 2 and lower levels, 5.5 (AUC $=.966 ; 95 \% \mathrm{CI}$, .943-.989); between FAC 3 and lower levels, 11.5 (AUC $=.979 ; 95 \% \mathrm{CI}, .962-.995)$; between FAC 4 and lower 
Table 4. Cutoff Value of the FGA According to the FAC

\begin{tabular}{ccccc}
\hline FAC & Cutoff value & Sensitivity & Specificity & AUC (95\% CI) \\
\hline FAC $\geq 1$ from FAC 0 & .5 & .906 & 1.000 & $.953(.926$ to .980$)$ \\
FAC $\geq 2$ from FAC $\leq 1$ & 5.5 & .919 & .919 & $.966(.943$ to .989$)$ \\
FAC $\geq 3$ from FAC $\leq 2$ & 10.5 & .940 & .926 & $.979(.962$ to .995$)$ \\
FAC $\geq 4$ from FAC $\leq 3$ & 14.5 & .881 & .881 & $.954(.929$ to .980$)$ \\
FAC 5 from FAC $\leq 4$ & 18.5 & .864 & .867 & $.959(.935$ to .983$)$ \\
\hline
\end{tabular}

FGA: Functional Gait Analysis; FAC: Functional Ambulation Category; FAC 0 = nonfunctional ambulator; FAC $1=$ dependent on physical assistance(level 2, continuous manual contact); FAC 2 = dependent on physical assistance(level 1, intermittent touch); FAC 3 = dependent on supervision; FAC 4 = independent, level surface only; FAC 5 = independent, everywhere.
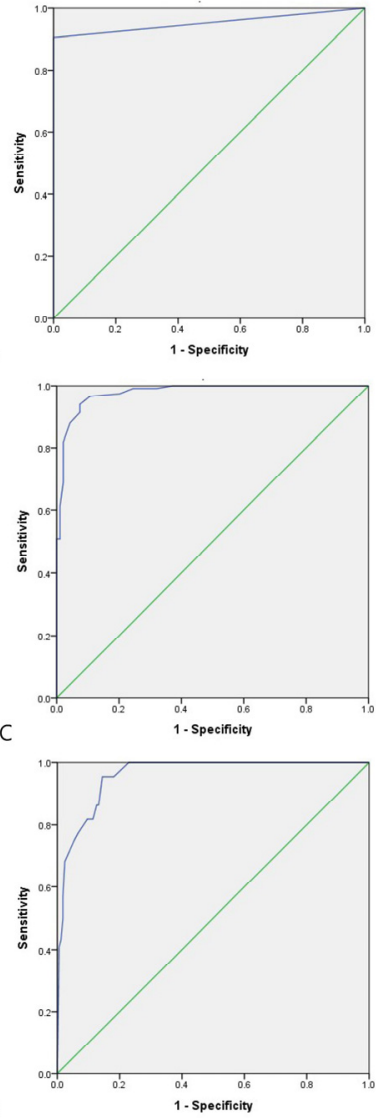

B
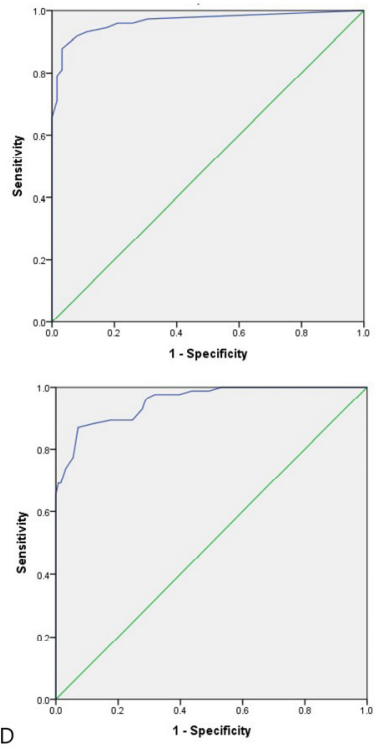

Fig. 1. Receiver operating characteristic curves. (A) between FAC 1 and FAC 0; (B) between FAC 2 and lower levels; (C) between FAC 3 and lower levels; (D) between FAC 4 and lower levels; and (E) between FAC 5 and lower levels.

levels, 14.5 (AUC = .954; 95\% CI, .929-.980); and between

FAC 5 and lower levels, $18.5(\mathrm{AUC}=.959 ; 95 \% \mathrm{CI}$, .935-.983) (Table 4) (Fig. 1).
5. Stepwise multiple linear regression on the individual FGA items

The individual FGA items selected for stepwise multiple 
Table 5. Stepwise Linear Regression Analysis of the FGA Items

\begin{tabular}{cccccc}
\hline Source & $\mathrm{R}^{2}$ & Beta & $\mathrm{t}$ & $\mathrm{p}$-Value & VIF \\
\hline Overall model & .86 & & & & \\
Item 10 & & .403 & 6.957 & .000 & 4.884 \\
Item 2 & .226 & 3.159 & .002 & 7.441 \\
Item 3 & .160 & 2.504 & .013 & 5.948 \\
Item 1 & .188 & 2.482 & .014 & 8.367 \\
\hline
\end{tabular}

FGA: Functional Gait Analysis; VIF: Variance Inflation Factor; Item 1 = gait level surface; Item 2 = change in gait speed; Item 3 = gait with horizontal head turns; Item $10=$ steps.

regression were items 1,2,3, and 10. Approximately $86 \%$ of the variance in the overall FAC scores could be explained by the scores on these items, which are as follows in order of the variance: item $10(\mathrm{~B}=.403), 2(\mathrm{~B}=.226), 1$ ( $\mathrm{B}$ $=.188)$, and $3(\mathrm{~B}=.160)$. Items $4(\mathrm{p}=.586), 5(\mathrm{p}=.354)$, $6(\mathrm{p}=.969), 7(\mathrm{p}=.110), 8(\mathrm{p}=.272)$, and $9(\mathrm{p}=.825)$ were excluded by the stepwise regression analysis (Table 5).

\section{Discussion}

This study examined which OGA tools best measure the independence of gait, as defined by the FAC, in hemiparetic stroke patients. The FGA score could discriminate among the independence, supervision, and dependence aspects of gait. Thus, an improving FGA score may be an effective means of improving the gait independence in stroke patients.

The results also showed that the total FGA score was associated with the level of gait independence. The FGA can be used as a reliable and valid tool to assess the functional gait performance in patients after stroke, including balance during walking [26]. Frequent balance disturbances during walking are one of the most important risk factors for falls [27]. Patients with balance problems while walking are more dependent [28]. Thieme et al. also found a strong correlation between the FGA scores and FAC classifications. FAC measures the gait ability, which corresponds to the degree of requirement for assistance with gait. The use of walking aids, such as a cane, does not limit the applicability of the FAC, which is a general tool covering a variety of related functions. Moreover, the high correlation of FAC classifications with FGA scores demonstrates the impact of walking balance on gait ability. Furthermore, the results showed significant differences in FGA scores according to the FAC classification. For participants at FAC level 3, the mean FGA score was 7, whereas mean scores of 14 and 23 were noted for those at FAC levels 4 and 5 , respectively [26]. The cutoff values identified in the study can be used to determine the ambulation safety, prescribe assistive devices, and establish treatment goals [29]. Wrisley et al. reported that an FGA cutoff score of 22 was effective in classifying the fall risk and predicting unexplained falls in community-dwelling older adults [30]. Leddy et al. reported that a score of 15 on the FGA had predictive value for clinically identifying fallers among Parkinson's patients [31]. The present study identified the cutoff FGA scores for FAC levels 1-5 of .5, $5.5,11.5,14.5$, and 18.5 points, respectively. The study findings suggest a lower probability of ceiling effects for the FGA than reported in previous studies. Therefore, the FGA cutoff values for each FAC level appear to be useful indicators for treatment and evaluation, emphasizing the independent gait in stroke patients.

This study also evaluated other common assessment tools: WGS, TGS, and RVGA. On the other hand, the total scores for these tools were not associated using the 
FAC classifications. Observational gait assessment scales, such as the TGS, WGS, RVGA, and the Gait Assessment and Intervention Tool (GAIT), were designed to evaluate the deviations from normal gait patterns, as were scales that explicitly assess the walking disability, such as the Functional Ambulation Categories and the Dynamic Gait Index [13]. The WGS, which was designed by Rodriquez et al., is a visual gait assessment tool that evaluates 14 observable temporal gait parameters and specific body movement patterns, such as those associated with the use of a hand-held gait aid, as well as stance time and width, weight shift, toe-off, hip, knee, and pelvic motions, and heel strike of the affected leg [32]. Because the WGS quantifies the hemiplegic gait quality, it is sensitive to all possible modifications of the gait pattern [21]. The TGS includes items that assess only the temporal aspects of gait (e.g., step length) and compensatory strategies, such as gait asymmetry and step discontinuity [9]. A visual gait assessment using the RVGA is more reliable when global scores alone are used to evaluate abnormal gait patterns [23]. These tools primarily assess the spatial and temporal aspects of the gait pattern. Thus, their results reflect the gait patterns that do not significantly affect the independence in walking. Other functions, such as balance, have a greater effect on gait independence.

The present study determined the items on the FGA that were most closely associated with independence in walking. The results showed that items $1,2,3$, and 10 were most closely related to the FAC level. Item 1 addressed the gait on a level surface, and item 2 dealt with changes in gait speed. Viosca et al. reported a significant correlation among the functional classification levels, walking velocity, and the number of steps taken, suggesting that the present functional classification is valid because it classifies subjects according to their walking ability, and it is useful for assessing the recovery of gait [8]. Lower FGA scores were correlated with a slower gait speed, and other measures of imbalance and frailty that may influence a person's mobility and ultimately increase the risk of falling or other consequences of decreased mobility [33]. Kollen et al. found that the FAC score was correlated with the walking velocity [34]. Several authors also considered the walking velocity to provide information on patient recuperation, the treatment efficiency, and the patient's degree of functional independence $[35,36]$. Item 3 assesses the gait during horizontal head turns. Voluntary head motions during standing or walking are performed frequently during daily activities [37]. The FGA incorporates many related tasks, such as the ability to walk with head movement, turn safely, and walk backward. The ability to perform these tasks is necessary for functional mobility in daily life. Difficulty with these tasks may suggest specific interventions directed toward improving the functional gait, such as walking with head turns on various surfaces or provision of education regarding compensatory techniques [30]. Item 10 assesses the ability to navigate steps. The stair mobility is key to a successful return to the community, as managing stairs is a significant determinant of independence and active community living [38]. ShumwayCook et al. reported the importance of stair ambulation in maintaining independence and activity in the community [39]. The results of the present study showed that the gait independence of stroke patients varied depending on their ability to navigate stairs. These results support previous research findings indicating that better stair mobility is associated with independent walking.

This study had some limitations. First, the subjects were in-patients of a hospital rehabilitation ward. Therefore, it is unclear if the derived cutoff values can be applied to community-based stroke patients. In addition, this study did not evaluate all available gait assessment tools. Future studies must consider examining the gait independence in stroke patients using other OGA tools. Furthermore, the study should consider individual items on the tools in more detail for a more thorough evaluation of the independence of gait of stroke patients. Finally, a future study needs 
to identify the reliability evaluation of each observational tool to the functional gait performance.

\section{Conclusions}

FGA can be used to assess the level of gait independence after stroke, and therapists may find it helpful as an evaluation tool. In addition, therapists can apply the FGA total score cutoff values defined herein for determining the level of assistance required by a patient undergoing rehabilitation for walking. FGA items 1, 2, 3, and 10 were particularly relevant to the independent gait in stroke patients. These items are especially useful in rehabilitation efforts aimed at independent walking in stroke patients.

\section{References}

[1] Chiou II, Burnett CN. Values of activities of daily living. A survey of stroke patients and their home therapists. Phys Ther. 1985;65:901-6.

[2] von Schroeder HP, Coutts RD, Lyden PD, Billings E Jr, Nickel VL. Gait parameters following stroke: a practical assessment. J Rehabil Res Dev. 1995;32: 25-31.

[3] Wade DT, Hewer RL. Functional abilities after stroke: measurement, natural history and prognosis. J Neurol Neurosurg Psychiatry. 1987;50:177-82.

[4] Perry J, Garrett M, Gronley JK, Mulroy SJ. Classification of walking handicap in the stroke population. Stroke. 1995;26:982-9.

[5] Lord SE, McPherson K, McNaughton HK, Rochester L, Weatherall M. Community ambulation after stroke: how important and obtainable is it and what measures appear predictive? Arch Phys Med Rehabil. 2004;85: 234-9.

[6] van de Port IG, Kwakkel G, Lindeman E. Community ambulation in patients with chronic stroke: how is it related to gait speed? J rehabil Med. 2008;40:23-7.

[7] Dobkin BH. Training and exercise to drive poststroke recovery. Nat Clin Pract Neurol. 2008;4:76-85.

[8] Viosca E, Martínez JL, Almagro PL, Gracia A, González C. Proposal and validation of a new functional ambulation classification scale for clinical use. Arch Phys Med Rehabil. 2005;86:1234-8.

[9] Zimbelman J, Daly JJ, Roenigk KL, Butler K, Burdsall R, Holcomb JP. Capability of 2 gait measures for detecting response to gait training in stroke survivors: Gait Assessment and Intervention Tool and the Tinetti Gait Scale. Arch Phys Med Rehabil. 2012;93:129-36.

[10] Gronley JK, Perry J. Gait analysis techniques: Rancho Los Amigos Hospital gait laboratory. Phys Ther. 1984; 64:1831-8.

[11] Bethoux F, Bennett S. Evaluating walking in patients with multiple sclerosis: which assessment tools are useful in clinical practice? Int J MS Care. 2011;13:4-14.

[12] Van Bloemendaal M, van de Water AT, van de Port IG. Walking tests for stroke survivors: a systematic review of their measurement properties. Disabil Rehabil. 2012;34:2207-21.

[13] Gor-García-Fogeda MD, Cano de la Cuerda R, Carratalá Tejada M, Alguacil-Diego IM, Molina-Rueda F. Observational Gait Assessments in People With Neurological Disorders: A Systematic Review. Arch Phys Med Rehabil. 2016:97:131-40.

[14] Coutts F. Gait analysis in the therapeutic environment. Man Ther. 1999;4:2-10.

[15] Eastlack ME, Arvidson J, Snyder-Mackler L, et al. Interrater reliability of videotaped observatioinal gaitanalysis assessment. Phys Ther. 1991;71:465-72.

[16] Wrisley DM, Marchetti GF, Kuharsky DK, Whitney SL. Reliability, internal consistency, and validity of data obtained with the functional gait assessment. Phys Ther. 2004:84:906-18.

[17] Wrisley DM, Walker ML, Echternach JL, Strasnick B. Reliability of the dynamic gait index in people with vestibular disorders. Arch Phys Med Rehabil. 2003:84: 1528-33. 
[18] Holden MK, Gill KM, Magliozzi MR, Nathan J, PiehlBaker L. Clinical gait assessment in the neurologically impaired. Reliability and meaningfulness. Phys Ther. 1984;64:35-40.

[19] Wade DT. Measurement in neurological rehabilitation. New York. Oxford: Oxford Medical Publications. 1992.

[20] Mehrholz J, Wagner K, Rutte K, Meissner D, Pohl M. Predictive validity and responsiveness of the functional ambulation category in hemiparetic patients after stroke. Arch Phys Med Rehabil. 2007;88:1314-9.

[21] Brock KA, Goldie PA, Greenwood KM. Evaluating the effectiveness of stroke rehabilitation: choosing a discriminative measure. Arch Phys Med Rehabil. 2002;83:92-9.

[22] Salter K, Campbell N, Richardson M, Mehta S, Jutai $\mathrm{J}$, Zettler L, et al. Outcome measures in stroke rehabilitation. The evidence-based review of stroke rehabilitation (EBRSR) reviews current practices in stroke rehabilitation. 2013. Qvailable at http://www.ebrsr.com /evidence-review/20-outcome-measures-stroke-rehabilit ation. Accessed November 1, 2021.

[23] Lord SE, Halligan PW, Wade DT. Visual gait analysis: the development of a clinical assessment and scale. Clin Rehabil. 1998;12:107-19.

[24] Pizzi A, Carlucci G, Falsini C, Lunghi F, Verdesca S, Grippo A. Gait in hemiplegia: evaluation of clinical features with the Wisconsin Gait Scale. J Rehabil Med. 2007;39:170-4.

[25] Tinetti ME. Performance-oriented assessment of mobility problems in elderly patients. J Am Geriatr Soc. 1986;34: 119-26.

[26] Thieme H, Ritschel C, Zange C. Reliability and validity of the functional gait assessment (German version) in subacute stroke patients. Arch Phys Med Rehabil. 2009;90:1565-70.

[27] Lamb SE, Ferrucci L, Volapto S, Fried LP, Guralnik JM; Women's Health and Aging Study. Risk factors for falling in home-dwelling older women with stroke: the Women's Health and Aging Study. Stroke. 2003;34:
494-501.

[28] Tyson SF, Hanley M, Chillala J, Selley A, Tallis RC. Balance disability after stroke. Phys Ther. 2006;86:30-8.

[29] Fujita T, Sato A, Yamamoto Y, Otsuki K, Tsuchiya K, Tozato F. Motor Function Cutoff Values for Independent Dressing in Stroke Patients. Am J Occup Ther. 2016;70: 1-7.

[30] Wrisley DM, Kumar NA. Functional gait assessment: concurrent, discriminative, and predictive validity in community-dwelling older adults. Phys Ther. 2010;90: 761-73.

[31] Leddy AL, Crowner BE, Earhart GM. Functional gait assessment and balance evaluation system test: reliability, validity, sensitivity, and specificity for identifying individuals with Parkinson disease who fall. Phys Ther. 2011;91:102-13.

[32] Rodriquez AA, Black PO, Kile KA, et al. Gait training efficacy using a home-based practice model in chronic hemiplegia. Arch Phys Med Rehabil. 1996;77:801-5.

[33] American Geriatrics Society, British Geriatrics Society, and American Academy of Orthopedic Surgeons Panel on Falls Prevention. Guideline for the prevention of falls in older persons. J Am Geriatr Soc. 2001;49:664-72.

[34] Kollen B, Kwakkel G, Lindeman E. Time dependency of walking classification in stroke. Phys Ther. 2006;86: 618-25.

[35] Robinett CS, Vondran MA. Functional ambulation velocity and distance requirements in rural and urban communities. A clinical report. Phys Ther. 1988;68: 1371-3.

[36] Bohannon RW. Gait performance of hemiparetic stroke patients: selected variables. Arch Phys Med Rehabil. 1987;68:777-81.

[37] Lamontagne A, Paquet N, Fung J. Postural adjustments to voluntary head motions during standing are modified following stroke. Clin Biomech. 2003;18:832-42.

[38] Alzahrani MA, Dean CM, Ada L. Ability to negotiate stairs predicts free-living physical activity in community- 
dwelling people with stroke: an observational study. Aust J Physiother. 2009;55:277-81.

[39] Shumway-Cook A, Patla AE, Stewart A, Ferrucci L, Ciol MA, Guralnik JM. Environmental demands associated with community mobility in older adults with and without mobility disorders. Phys Ther. 2002;82: 670-81. 